\title{
Alltagsgespräche im Kindergarten - Gelegenheitsstrukturen für den Erwerb bildungssprachlicher Fähigkeiten
}

\section{Dieter Isler, Sibylle Künzli und Esther Wiesner}

Im Projekt "Prozesse der Sprachförderung im Kindergarten (ProSpik)»1 werden Gespräche zwischen Lehrpersonen und Kindern gefilmt und sequenzanalytisch ausgewertet, um ibre Potenziale für den Erwerb und die Förderung bildungssprachlicher Fähigkeiten zu untersuchen. Ziel ist es, Grundlagen für eine stufengerechte (integrierte, situations- und themenorientierte) Sprachdidaktik zu erarbeiten, die Bildungsungleichheit nicht reproduziert, sondern zu ibrem Abbau beiträgt. Der vorliegende Beitrag informiert über die Grundlagen und die Anlage des Projekts sowie über den Arbeitsstand nach einem Jahr. Im ersten Abschnitt geht es um Sprache und Bildungsungleichheit, den Kindergarten in der Deutschschweiz und um Sprachförderung 4- bis 6-jähriger Kinder. Im zweiten Abschnitt wird das Projekt vorgestellt und theoretisch sowie methodologisch verortet. Der dritte Abschnitt gibt einen exemplarischen Einblick in die Auswertungsarbeiten. Abgeschlossen wird der Beitrag mit einer Verortung der Ergebnisse im Tagungsthema.

\section{Ausgangs lage}

Bildungsungleichheit und frühe Bildung

In der Schweiz besteht bereits beim Schuleintritt ein vergleichsweise starker Zusammenhang zwischen familiären Bedingungen und sprachlichen Fähigkeiten der Kinder (Moser, 2005; Stamm, 2012). Studien aus Deutschland bestätigen diesen Zusammenhang bereits für 3- und 4-jährige Kinder (Niklas, Schmiedeler \& Schneider, 2010; Weinert, Ebert \& Dubowy, 2010). Wie Forschungsergebnisse aus Grossbritannien und Deutschland zeigen, kann der Besuch qualitativ guter vorschulischer Bildungseinrichtungen einen nachhaltigen Beitrag zur Verbesserung der Bildungschancen benachteiligter Kinder leisten (Bos et al., 2003; Sammons et al., 2008). Daher ruhen viele Hoffnungen auf der frühen Bildung im Allgemeinen und auf der frühen Sprachförderung im Besonderen (OECD, 2010). Es wird jedoch auch vor überzogenen Erwartungen und Aktivismus gewarnt (Grossenbacher, 2007): Erstens, weil pädago- 
gische Einrichtungen den Erwerb sprachlicher Fähigkeiten im Vergleich zu den familialen Kontexten weniger stark beeinflussen (Stamm, 2012; Sylva et al., 2011), und zweitens, weil heute über Bildung im Früh- und Vorschulbereich, Gelingensbedingungen sprachlicher Förderung, Professionalisierung des Fachpersonals und Mechanismen des Abbaus von Bildungsungleichheit noch kaum gesicherte Erkenntnisse vorliegen (Ehlich, 2005; Fried, 2010; Roßbach, Klugcniok \& Kuger, 2008).

\section{Der Kindergarten in der Deutschschweiz}

Der Kindergarten durchläuft in der Deutschschweiz seit gut zwei Jahrzehnten einen tiefgreifenden institutionellen Wandel und entwickelt sich von einer sozialpädagogischen Betreuungs- zu einer schulischen Bildungseinrichtung (Sörensen \& Thévenaz-Christen, 2003). ${ }^{2}$ Während für die Schule schon lange die Kantone zuständig sind, galt für den Kindergarten bis vor kurzem eine kommunale Gebundenheit (ebd.). Heute sind (mit Ausnahme des Kantons Wallis) die Kantone für den Kindergarten zuständig. Im Schuljahr 2012/2013 besuchte die grosse Mehrheit der Kinder den Kindergarten zwei Jahre lang und auch in Kantonen mit einjähriger oder fehlender Besuchspflicht hat sich der zweijährige Besuch durchgesetzt (EDK, 2013). Daneben haben sich weitere strukturelle Rahmenbedingungen des Kindergartens in den letzten 10 bis 15 Jahren stark verändert: Organisatorisch sind Kindergärten heute in der Regel in Schuleinheiten integriert, in vielen Kantonen sind Lehrpläne für den Kindergarten in Kraft, und die Lehrpersonen für den Kindergarten werden an Pädagogischen Hochschulen ausgebildet. Damit hat sich der Kindergarten in der Deutschschweiz als öffentliche, unentgeltliche und flächendeckende zweijährige Bildungseinrichtung für 4- bis 6-Jährige etabliert. Dieser institutionelle Wandel wird durch die laufenden Bestrebungen zur Harmonisierung des Schweizer Schulsystems weiter verfestigt: Im bereits eingeführten Plan d'étude romand (CIIP, 2008) wie auch im vor der Einführung stehenden Lehrplan 21 (D-EDK, 2013) werden die beiden Kindergartenjahre und die 1. und 2. Schuljahre zu einem vierjährigen Zyklus zusammengefasst.

Sprachliche Fähigkeiten werden im Kindergarten traditionellerweise nicht im Rahmen spezifischer Unterrichtsgefässe, sondern themen- und situationsorientiert und dem Entwicklungsstand der Kinder angepasst gefördert (Witzig, 2002). Aktivitäten wie Geschichten hören und erfinden, Erlebnisse erzählen oder Verse aufsagen spielen bei der Sprachförderung eine zentrale Rolle (Erziehungsdirektion des Kantons Bern, 1999). Mit der schrittweisen Einbindung des Kindergartens in das Schulsystem und unter dem Einfluss der bildungspolitischen Fokussierung auf frühe Bildung und sprachliche Fähigkeiten verändert sich aber auch das Verständnis von Sprachförderung im Kindergarten. Der Lehrplan 21 (D-EDK, 2013) ist durchgängig nach Fachbereichen strukturiert, und die Beschreibungen der schulsprachlichen Kompetenzen für den gesamten ersten Zyklus sind dem Fach Deutsch zugeordnet (ebd.). Dieser Wandel von 
einer stufen- zu einer fachdidaktischen Orientierung zeigt sich auch auf der Ebene der didaktischen Modelle und Hilfsmittel für den Unterricht: In den letzten zehn Jahren wurde für die Sprachförderung 4- bis 6-jähriger Kinder eine Vielzahl von Diagnoseinstrumenten, Förderprogrammen und Lehrmitteln publiziert (für die Schweiz u.a. Aellig, Alt \& Bass, 2009; Baumann Schenker, Knechtel \& Kruse, 2010; Bitter Bättig et al., 2012; Moser \& Berweger, 2008; Niedermann \& Sassenroth, 2002). Viele Instrumente sind auf einzelne sprachliche Fertigkeiten wie Wortschatz, phonologische Bewusstheit oder Buchstabenkenntnis ausgerichtet, während der Erwerb komplexer sprachlicher Handlungsfähigkeiten wie Erzählen, Erklären oder Argumentieren nicht gezielt unterstützt wird (Fried, 2008). In dieser Situation erscheint es sinnvoll, die Sprachförderpotenziale des Kindergartenalltags zu untersuchen und damit zur Entwicklung einer stufengerechten Sprachdidaktik für den ersten Zyklus der schulischen Bildung beizutragen.

\section{Sprachförderung 4- bis 6-jähriger Kinder}

Im hier vorgestellten Projekt werden Prozesse der Sprachförderung im Kindergarten untersucht. Deshalb interessiert an dieser Stelle der Forschungsstand zur sprachlichen Förderung 4- bis 6-jähriger Kinder in Bildungseinrichtungen. Wie die internationale Forschung zeigt, spielt die Qualität von Bildungsprozessen im Unterricht eine Schlüsselrolle bei der vorschulischen Förderung sprachlicher Fähigkeiten (Henry \& Pianta, 2011; Kuger \& Kluczniok, 2008). Für Zusammenhänge zwischen dem Handeln der pädagogischen Fachpersonen und sprachlichen Fähigkeiten der Kinder gibt es verschiedene Belege: Kinder sind sprachlich aktiver, wenn Lehrpersonen selber oft und kompetent sprechen, die Kinder zum Selbersprechen ermuntern und sie beim Erwerb neuer Wörter unterstützen (Farran, Aydogan, Khan \& Lipsey, 2006). Herausfordernde Gespräche beim Bücherlesen, die Verwendung von gesprächsanregenden und modellierenden Strategien, Gespräche über Wortbedeutungen und Kontakt zu seltenen Wörtern begünstigen den Wortschatzerwerb (Vasilyeva \& Waterfall, 2011). Die Verwendung syntaktisch komplexer Sätze durch die Lehrperson steht in einem deutlichen Zusammenhang mit den Leistungen der Kinder im Bereich der syntaktischen Komplexität (ebd.). Diese Befunde belegen, dass das Handeln der Lehrpersonen in Gesprächen die sprachliche Produktivität sowie den Wortschatz- und Syntaxerwerb der Kinder unterstützen kann.

Aus dem deutschen Sprachraum liegen einige Studien vor, in welchen Prozesse der Sprachförderung direkt beobachtet wurden. Gasteiger-Klicpera, Knapp und Kucharz (2010) haben Sprachförderkurse für Vorschulkinder gefilmt. Die Ergebnisse zeigen, dass bezüglich verschiedener Qualitätsmerkmale der Sprachförderung (Expansion der Kinderäusserungen, Verteilung der Gesprächsanteile, Einbettung des Wortschatzes in einen Satzkontext und Reagieren auf Fehler) deutliche Unterschiede bestehen, und zwar nicht nur fallvergleichend zwischen den Förderlehrpersonen, sondern auch fallintern zwischen verschiedenen Situa- 
tionen (Gasteiger-Klicpera, Knapp \& Kucharz, 2010). Dabei können unterschiedliche, auch organisatorische Situationen für die Sprachförderung genutzt werden (ebd.). König (2010) hat Erzieherinnen in deutschen Kindergärten in ihrem beruflichen Alltag gefilmt und deren sprachlich-interaktionales Handeln untersucht. Die pädagogischen Fachpersonen beteiligen sich (mit abnehmender Häufigkeit) reagierend, abwartend/zuhörend, iniziierend/nachspürend, erweiternd, sprachfrei handelnd, delegierend und erst an letzter Stelle dialogisch entwickelnd an Gesprächen mit Kindern (König, 2010). Die Autorin schliesst daraus, «dass die konkrete Interaktion im Kindergarten derzeit noch nicht genutzt wird, um Lern- und Bildungsprozesse anzuregen», und fordert deshalb «eine Didaktik, die den PädagogInnen konkrete Hinweise gibt, wie Lern- und Bildungsprozesse gut unterstützt werden können» (ebd. S. 351). Die Analysen dieser beiden Studien waren beschreibend auf die Häufigkeit bestimmter Interaktionsmerkmale ausgerichtet. Der Prozess der interaktiven Sinn-Ko-Konstruktion blieb dabei zwangsläufig ausgeblendet.

Aus der psycholinguistischen Gesprächsforschung liegen mehrere aktuelle Studien vor, die spezifische Sprachhandlungen bzw. global strukturierte Diskurseinheiten (Quasthoff et al., 2011) in Interaktionen von pädagogischen Fachpersonen und Kindern untersucht haben. ${ }^{3}$ Morek (2012) hat in zwei ersten Grundschulklassen Frontalunterricht und Gesprächskreise gefilmt und Situationen des Erklärens untersucht (Morek, 2012). Erkläraktivitäten werden vorwiegend durch Lehrerinnen initiiert, wobei zwei Hauptformen unterschieden werden können: Erstens das orchestrierte Erklären, welches von der Lehrerin gesteuert wird, möglichst viele Kinder einbezieht, nur lokale Zugzwänge schafft und der gemeinsamen Sinn-Ko-Konstruktion im Klassenkollektiv dient. Zweitens das solistische Erklären bei welchem einzelne Kinder vor- oder eingeladen werden eine längere Erklärung zu produzieren (ebd.). Das solistische Erklären kann funktional eher der inhaltlichen Verständigung dienen, wobei Kinder mit entsprechendem Wissen die Rolle der Expertin oder des Experten übernehmen. Alternativ dient es eher der formalen Erledigung einer von der Lehrerin gestellten Aufgabe in der Rolle der Schülerin oder des Schülers (ebd.). Die beiden untersuchten Lehrerinnen unterscheiden sich bezüglich des Vorkommens formbzw. funktionsorientierter Formen des Erklärens und bei der Realisierung der damit verbundenen interaktiven Aufgaben. Dabei zeigt sich, «dass institutionelle Kommunikation, wenngleich sie sich stets unter bestimmten Bedingungen vollzieht, im und durch den Vollzug des sprachlichen Handelns (...) unterschiedlich gestaltet werden kann.» (ebd. S. 274).

Heller (2012) hat in vier ersten Grundschulklassen Unterrichtslektionen gefilmt und im Hinblick auf (u.a.) Interaktionsmuster der Lehrpersonen und die interaktive Herstellung von Passung und Divergenz untersucht. Die Fälle unterscheiden sich deutlich: Lehr-Lerndiskurse werden in zwei Klassen als Wissenskonstruktionen, in den beiden anderen dagegen als Wissensdemonstrationen und -überprüfungen ausgestaltet. Das erste Interaktionsmuster eröffnet den Kindern unterschiedliche Beteiligungsmöglichkeiten als kompetente Problem- 
löser/innen. Das zweite bietet eher enge Antwortspielräume, erlaubt kaum Elaborierungen, Präzisierungen und Korrekturen und (re-)produziert durch normative Bewertungen gute und schlechte Schülerinnen und Schüler (ebd.). Was die Herstellung von Passung und Divergenz betrifft, lassen sich keine fallspezifischen Stile erkennen. Es zeigen sich aber dyadenspezifische Muster: Lehrperson betreiben je nach Kind mehr oder weniger interaktiven Aufwand, um das Erfüllen globaler Zugzwänge ${ }^{4}$ zu unterstützen (ebd.). Dadurch können sich Kinder als «non-members» der Lerngemeinschaft erfahren, verinnerlichen diese Rolle, reduzieren in der Folge ihre Beteiligung an Unterrichtsgesprächen und können diese nur noch eingeschränkt als Erwerbskontexte nutzen (ebd.). Damit legt die Autorin ein empirisch gestütztes Modell vor, welches die interaktive Herstellung von Bildungsungleichheit erklären kann.

Stude (2013) hat Gespräche von Erzieherinnen und etwas jüngeren (3- bis 6-jährigen) Kindern in Kindertagesstätten (sowie von Kindern untereinander) untersucht. Dabei ging es ihr um Gespräche als Erwerbskontexte metasprachlicher Fähigkeiten bzw. um Situationen, in welchen Sprache verbal-explizit thematisiert wurde (Stude, 2013). Die Studie zeigt, dass die untersuchten Erzieherinnen Sprache insbesondere im Hinblick auf den Erwerb und die Einhaltung kultur- und institutionenspezifischer Verhaltensnormen thematisieren und dabei nicht die (meta-)sprachliche Förderung, sondern die Erziehung der Kinder verfolgen (ebd.).

Auf dieser Grundlage lässt sich festhalten, dass kommunikative Praktiken von pädagogischen Fachpersonen in zweifacher Hinsicht bildungsrelevant sind: Erstens fungieren Interaktionsmuster von Lehrpersonen des Kindergartens als Erwerbskontexte sprachlicher Fähigkeiten unterschiedlicher Art. Zweitens sind Gespräche der Ort, an welchem sich herkunftsbedingte Passung bzw. Divergenz manifestiert, Rollen und Hierarchien interaktiv konstruiert werden und Ungleichheit reproduziert oder abgebaut wird. Im Hinblick auf weiterführende Forschungsarbeiten ergeben sich folgende Desiderate:

- Der Deutschschweizer Kindergarten unterscheidet sich institutionell und organisatorisch stark von deutschen Kindertagesstätten und Grundschulen. Sprachliche Praktiken müssen unter den spezifischen Bedingungen des Deutschschweizer Kindergartens untersucht werden, damit sie für dieses Praxisfeld nutzbar gemacht werden können.

- Die berichteten Studien beleuchten entweder allgemeine Qualitätsaspekte von pädagogischen Interaktionen oder einzelne Sprachhandlungen. Es fehlen Untersuchungen, die auf den Erwerb bildungssprachlicher Fähigkeiten ausgerichtet sind.

- Die referierten Studien beschränken sich auf kodierende, deskriptive Auswertungen oder analysieren nur die verbalen Anteile der Audio- bzw. Videodaten. Sinn-Ko-Konstruktionen können mit multimodalen Sequenzanalysen vollständiger rekonstruiert werden.

- Der theoretische Ansatz von Heller (2012) zur Modellierung der Herstellungsprozesse von Bildungsungleichheit sollte weiter ausgearbeitet werden. 


\section{Das Projekt "Prozesse der Sprachförderung im Kindergarten»}

\section{Untersuchungsgegenstand und Fragestellung}

Das hier vorgestellte Projekt knüpft an diese Desiderate an: Es untersucht Deutschschweizer Kindergärten als Erwerbskontexte bildungssprachlicher Fähigkeiten anhand von Sequenzanalysen audiovisueller Daten im Hinblick auf die Reproduktion bzw. den Abbau von Bildungsungleichheit. Verfolgt werden zwei Erkenntnisinteressen (Habermas, 1968): ein praktisches, das darauf ausgerichtet ist, die Sprachförderung im Kindergarten genauer zu verstehen und weiter zu entwickeln, sowie ein emanzipatorisches, um verborgene Mechanismen der Reproduktion von Bildungsungleichheit aufzudecken und zu einer kritisch-rationalen Pädagogik beizutragen. Da über den Gegenstand noch wenig bekannt ist, soll im Rahmen dieses Projekts die komplexe Wirklichkeit sprachlicher Erwerbsprozesse im institutionellen Alltag des Kindergartens erkundet, rekonstruiert und konzeptualisiert werden. Angestrebte Ergebnisse sind detaillierte Sequenz- und Fallanalysen sowie daten- und theorieverankerte Konzepte, die als Grundlagen für die Qualifizierung von Lehrpersonen, die Entwicklung von didaktischen Hilfestellungen und die Generierung von weiterführenden Hypothesen verwendet werden können. Um diese Ziele zu erreichen, wird der Gegenstand anhand folgender Fragestellung untersucht:

1. Welche kommunikativen Formen bildungssprachlichen Handelns sind im Kindergarten anzutreffen?

2. Wie werden sie von den Lehrpersonen und Kindern interaktiv hergestellt?

3. (Wie) wird im Rahmen solcher Interaktionen soziale Ungleichheit von den Lehrpersonen und Kindern hergestellt, aufrecht erhalten oder abgebaut?

\section{Bezugstheorien}

Um den Zusammenhang von Sprache und Bildungsungleichheit auf der Mikroebene der Bildungsprozesse zu untersuchen, werden Konzepte aus Soziologie, Linguistik und Lernpsychologie beigezogen. Die Komplexität des untersuchten Gegenstandes macht das „Überschreiten disziplinärer Grenzen unabdingbar“ (Thole, Göbel \& Milbrandt, 2013). Diese unterschiedlichen disziplinären Zugänge stellen aber auch eine anspruchsvolle Herausforderung dar.

Den Rahmen bildet Bourdieus Theorie von Habitus und Feld (Bourdieu, 2001). In diesem Verständnis ist Sprache ein wichtiger Aspekt des kulturellen Kapitals. Im Verlauf alltäglicher sozialer Handlungen erwerben Kinder bestimmte sprachliche Praktiken mit spezifischen Merkmalen auf allen Ebenen des Sprachsystems und des Sprachgebrauchs. Wenn Kinder sprachlich handeln, greifen sie auf ihre habitualisierten Praktiken zurück. Ob sie ihr kulturelles Kapital erfolgreich einsetzen können, wird weitgehend durch die Regeln des jeweiligen Feldes bestimmt. Die Schule bewertet gewisse Praktiken höher als andere, und die Vertrautheit bzw. Unvertrautheit mit der «schulischen Form» 
(Thévenaz-Christen, 2005) dürfte massgeblich zur Bildungsungleichheit von Kindern unterschiedlicher Herkunft beitragen.

Die schulische Bildungssprache unterscheidet sich in verschiedener Hinsicht von der Alltagssprache. Erstens hat sie im Unterricht häufig den Charakter des «öffentlichen» Sprechens und Zuhörens (Erard \& Schneuwly, 2005): Die Lehrperson interagiert mit einem Kollektiv, und die Schülerinnen und Schüler äussern sich vor dem Publikum ihrer MitschülerInnen. Zweitens müssen in der Schule oft räumlich und zeitlich distante, fiktionale oder abstrakte Sachverhalte sprachlich repräsentiert werden. Deshalb ist die schulische Bildungssprache typischerweise konzeptionell schriftlich strukturiert, wobei sie medial mündlich oder schriftlich realisiert werden kann (Koch \& Österreicher, 1994). Drittens ist Sprache nicht nur Medium, sondern auch Gegenstand des Unterrichts. Die Objektivierung von Sprache unterstützt den Aufbau eines sekundären, theoretisch-reflexiven Zugangs zur Sprache, zur Welt und zum eigenen Denken (Heath, 1983; Lahire, 1993; Wygotski, 1934[1986]). Bildungssprachliche Fähigkeiten werden auf dieser Grundlage als Ressourcen zur Realisierung von a) konzeptionell schriftlichen Sprachhandlungen im Medium der Mündlichkeit (wie Berichten, Erzählen, Anleiten, Erklären, Argumentieren), b) medial schriftlichen Sprachhandlungen (mit Verwendung von Symbolen und Schrift) und c) Sprache objektivierenden Handlungen gefasst (Isler 2014). ${ }^{5}$ Diese textuellen, pragmatischen und metalinguistischen Fähigkeiten können als verborgene Sprachbarrieren (Käsler, 2005) wirken, wenn sie im Kindergarten und in der Schule stillschweigend vorausgesetzt, aber nicht vermittelt werden.

Bildungssprachliche Fähigkeiten sind kulturelle Werkzeuge, die durch Modelllernen beim gemeinsamen Handeln mit kompetenten Anderen erworben werden (Tomasello, 1999; Wygotski, 1934 [1986]). Dabei spielt die Herstellung und Aufrechterhaltung geteilter Aufmerksamkeit im Rahmen von Ko-Konstruktionsprozessen eine Schlüsselrolle (Sylva et al., 2011). Das Gelingen dieser Prozesse wird durch Handlungsmuster (Formate) unterstützt, die wiederkehrende sprachliche Handlungssituationen konsistent strukturieren (Bruner, 1983 [2002]). Wenn Kinder mit Handlungsmustern des Kindergartens vertraut sind, können sie ihre Aufmerksamkeit besser auf inhaltliche und sprachliche Merkmale ausrichten (Künzli, Isler \& Leemann, 2010). ${ }^{6}$

\section{Erhebungs- und Auswertungsmethoden}

Die Herstellung und Aufrechterhaltung geteilter Aufmerksamkeit ist als Sinn-Ko-Konstruktion im gemeinsamen, schrittweisen Handlungsvollzug zu verstehen (Schütz, 1932 [1984]). Die kommunikativen Formen und ihr interaktiver Vollzug werden mit Bezug auf den kommunikativen Konstruktivismus empirisch, holistisch (das Ensemble aller Ausdrucksmittel berücksichtigend), sequenziell und situiert untersucht (Knoblauch, 2013). Dabei wird das Geschehen als koordinierte Zusammenarbeit verstanden, die auf bestehendem Wissen der Subjekte aufbaut und den Erwerb sowie die Routinisierung von 
Wissen ermöglicht (ebd.). Es geht im Kern darum, die interaktive Herstellung der Vollzugswirklichkeit (Garfinkel, 1967) zu rekonstruieren. ${ }^{7}$

Diesem Grundverständnis entsprechend wurde für ProSpiK ein verstehender und rekonstruierender Zugang gewählt: Dem Ansatz der fokussierten Ethnografie bzw. «Videografie» (Knoblauch, 2006) folgend wird in acht Klassen aus vier Deutschschweizer Kantonen und mit unterschiedlichen Zusammensetzungen der Kindergartenalltag während je einer Woche mit Videoaufnahmen und Feldnotizen dokumentiert. Zusätzlich werden Unterrichtskommentare der Lehrpersonen festgehalten sowie Informationen zur Berufsbiografie der Lehrpersonen und zu den familialen Bedingungen der Kinder erhoben. Diese Daten werden im Sinne einer offenen, zirkulären und schrittweise vertiefenden Forschungsstrategie (Strauss \& Corbin, 1996) ausgewertet: Für jeden Kindergarten wird zunächst ein Logbuch erstellt, um eine Übersicht über die kommunikativen Ereignisse zu gewinnen. Auf dieser Grundlage werden einzelne Ereignisse ausgewählt, transkribiert und in Datensitzungen unter Beizug ethnografischen Wissens sequenzanalytisch ausgewertet. Einschlägige Befunde werden als Memos dokumentiert, fallintern und fallübergreifend verglichen, zu Konzepten ausgearbeitet und im Datenmaterial sowie theoretisch verankert. Durch den Einbezug unterschiedlicher (verbaler und visueller) Datensorten werden die Ergebnisse trianguliert (Flick, 2000 [2008]). Verfahren des Theoretical Sampling ermöglichen die Validierung von Konzepten und das gezielte Verfolgen der Forschungsfragen anhand weiterer Daten (Strauss, 1990 [1994]).

\section{Einblick in den Forschungsprozess}

Zum heutigen Zeitpunkt liegen Zwischenergebnisse aus dem ersten Projektjahr bzw. aus vier Kindergartenklassen vor. In diesem Abschnitt wird am Beispiel des Phänomens „Wechsel zwischen Referenzräumen“ gezeigt, wie im Projekt ProSpiK Videodaten sequenzanalytisch ausgewertet und konzeptualisiert werden. Zunächst werden einige Kontextinformationen gegeben, danach wird ein Ausschnitt aus einem Kreisgespräch in zwei Schritten ausgewertet. Abschliessend werden die Ergebnisse dieser Analysen gebündelt.

\section{Kontextinformationen zur vorgestellten Sequenz}

Der Kindergarten, um den es hier geht, ist Teil einer grösseren Schuleinheit in einer Deutschschweizer Grossstadt. Die Familien in seinem Einzugsgebiet leben mehrheitlich in grossen Mietshäusern. Von den 20 Kindern der Klasse sprechen sieben zuhause Deutsch und zwölf eine andere Sprache. Die Klassenzusammensetzung lässt sich im Vergleich zum Deutschschweizer Durchschnitt als eher unterprivilegiert und sprachlich heterogen beschreiben. Die Sequenz, die im Folgenden untersucht wird, hat sich an einem Donnerstag im Anschluss 
an die "Znüni»-Pause abgespielt. ${ }^{8}$ Die erfahrene Lehrerin und 8 ältere (5- und 6-jährige) Kinder sitzen im Stuhlkreis. Es geht darum, ein Bilderbuch nachzuerzählen, das die Lehrerin zu Beginn dieser Woche vorgelesen und mit den Kindern besprochen hat.

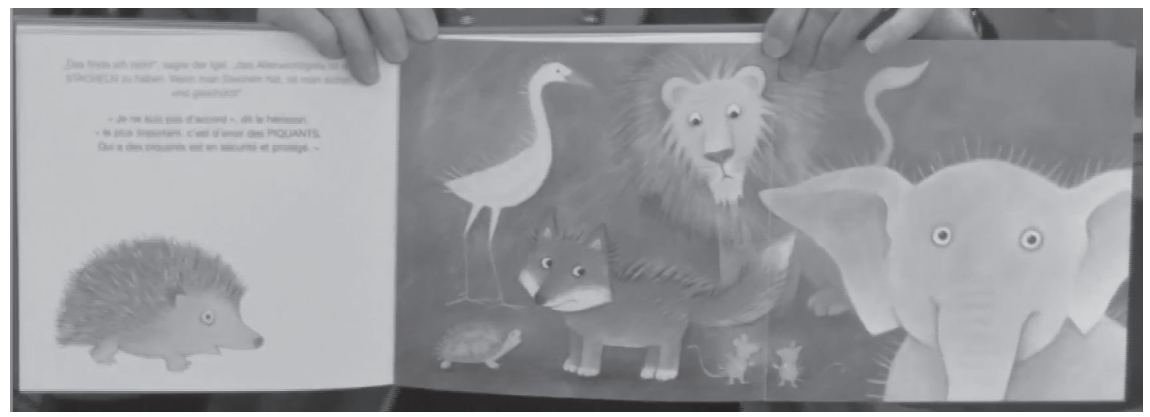

Abbildung 1: Doppelseite zur Igel-Episode aus dem Bilderbuch «Das Allerwichtigste» von Antonella Abbatiello (München: Hueber Verlag)

Die Geschichte besteht aus einzelnen Episoden, die sich auf jeder Doppelseite mit wechselnden Hauptrollen wiederholen: Verschiedene Tiere wetteifern im Gespräch um das allerwichtigste Körpermerkmal. In der fraglichen Szene plädiert der Igel für Stacheln als wichtigstes Attribut. Auf der weissen linken Buchseite ist der Igel abgebildet und sein Statement aufgeschrieben. Auf der bunten, aufgeklappten rechten Buchseite sind die zuhörenden Tiere mit Stacheln abgebildet.

Im Folgenden wird der Verlauf der Igel-Episode dargestellt und mit Blick auf das Phänomen «Wechsel zwischen Referenzräumen» ausgewertet. Die Darstellung erfolgt als zusammenfassende Paraphrasierung, weil sich das Phänomen «Wechsel der Referenzräume» auf einer mittleren Ebene der interaktiven Züge deutlicher veranschaulichen lässt als auf der Mikroebene der einzelnen Akte. ${ }^{9}$ Die Züge der Lehrperson werden in der linken, jene der Kinder in der rechten Spalte beschrieben. Die Episode wird in zwei Zugfolgen aufgeteilt. Jede Zugfolge wird zunächst im Überblick dargestellt und schrittweise erläutert, danach werden die Befunde gebündelt. Abschliessend werden die Erkenntnisse zum Phänomen «Wechsel der Referenzräume» und zu weiteren in dieser Episode manifesten Phänomenen zusammengefasst.

In der ersten Zugfolge geht es zunächst (in Zeile 1) um das Hier-und-Jetzt: das Auswählen des nächsten Kindes und die Übergabe des Buchs. Zusammen mit diesem Leitobjekt übergibt die Lehrerin Alena auch das Rederecht als primäre Sprecherin, und sie positioniert das Buch und seinen Inhalt als Gegenstand der geteilten Aufmerksamkeit. 
Zugfolge 1 der Igel-Episode

\begin{tabular}{|c|c|c|}
\hline $\begin{array}{l}\text { Die LP schlägt Alena }{ }^{8} \text { vor, die nächste Episode } \\
\text { zu erzählen, und gibt ihr das Buch. }\end{array}$ & 1 & \\
\hline & 2 & $\begin{array}{l}\text { Alena beginnt die Aussage des lgels zu } \\
\text { zitieren, bricht ab, überlegt und sagt, dass } \\
\text { sie es vergessen habe. }\end{array}$ \\
\hline \multirow{2}{*}{$\begin{array}{l}\text { Die LP geht weg, holt einen Stoff-lgel und } \\
\text { zeigt ihn Alena. }\end{array}$} & 3 & \\
\hline & 4 & Alena vervollständigt das Zitat des Igels. \\
\hline \multirow{2}{*}{$\begin{array}{l}\text { Die LP bestätigt Alenas Aussage und fragt sie, } \\
\text { wer das gesagt habe. }\end{array}$} & 5 & \\
\hline & 6 & Alena gibt die gesuchte Antwort (der lgel). \\
\hline \multirow{2}{*}{$\begin{array}{l}\text { Die LP bestätigt Alenas Antwort und fragt sie } \\
\text { nach der Begründung für die Aussage des Igels. }\end{array}$} & 7 & \\
\hline & 8 & $\begin{array}{l}\text { Alena setzt zu einer Begründung an (mit } \\
\text { "weil"), bricht ab, schaut auf die Buchseite } \\
\text { und sagt, dass sie es vergessen habe. }\end{array}$ \\
\hline \multirow{2}{*}{$\begin{array}{l}\text { Die LP fragt Alena mit gesenkter Stimme nach } \\
\text { dem Verhalten des Igels bei Gefahr. }\end{array}$} & 9 & \\
\hline & 10 & Alena gibt eine Antwort (wegrennen). \\
\hline $\begin{array}{l}\text { Die LP bestätigt Alenas Antwort, signalisiert } \\
\text { weitere mögliche Antworten und ruft Norena auf. }\end{array}$ & 11 & \\
\hline
\end{tabular}

Abbildung 2: Igel-Episode, 1. Zugfolge. Unterstrichen = auf das gemeinsame Handeln im Hier-und-Jetzt bezogen, kursiv = auf den Inhalt des Bilderbuchs bezogen, unmarkiert $=$ auf Weltwissen bezogen; $\mathbf{f e t t}=$ mit Objekten praktisch handelnd

Alena versteht diese Erwartungen und versucht sie zu erfüllen: Sie beginnt (in Zeile 2) sofort zu sprechen und bezieht sich thematisch auf den Inhalt des Buchs (die Aussage des Igels). Damit vollzieht sie den von der Lehrerin vorbereiteten Wechsel vom Hier-und-Jetzt zur Buchwelt. Allerdings fehlt ihr zur erwartungsgemässen Realisierung ihres Zugs das benötigte Wissen (die vollständige Aussage des Igels). Sie wechselt in dieser Situation auf die metakommunikative Ebene und kann damit ihren Zugzwang zumindest diskursiv erfüllen und das Rederecht wieder an die Lehrerin zurückgeben. Diese reagiert (in Zeile 3) nicht mit einer Entlassung von Alena aus der Rolle der primären Sprecherin, sondern mit einem nonverbalen Unterstützungsangebot: Sie holt einen Stoff-Igel und bietet ihn Alena als Hilfe zur Lösungsfindung an. Mit der Wiedergabe des Zitats (in Zeile 4) kann Alena die an sie gestellte Erwartung nun auch inhaltlich erfüllen. Damit ist die Lehrerin wieder am Zug. Sie evaluiert (in Zeile 5) Alenas Antwort positiv, entlässt sie aber noch nicht aus ihrer Rolle, sondern stellt ihr eine Anschlussfrage (nach der Identität des sprechenden Tieres). Alena kann diese Erwartung (in Zeile 6) diskursiv und inhaltlich erfüllen. Die Lehrerin stellt (in Zeile 7) eine weitere Anschlussfrage (nach der Begründung der Aussage des Igels). Erneut schliesst Alena (in Zeile 8) mit ihrem Zug sofort diskursiv passend an («Weil ...»), hat aber das erforderlichen Wissen nicht verfügbar und wechselt 
auf die metakommunikative Ebene. Diesmal verwendet die Lehrerin (in Zeile 9) eine verbale Unterstützungsstrategie: Sie lenkt Alenas Aufmerksamkeit vom Referenzraum der Buchwelt auf jenen des Weltwissens. Alena vollzieht diesen Wechsel nach und gibt (in Zeile 10) eine mögliche Antwort, die zwar sachlich korrekt ist, aber nicht mehr in Bezug zur Buchwelt steht (im Buch begründet der Igel sein Argument damit, dass er durch die Stacheln vor Feinden geschützt sei). Die Lehrerin entlässt Alena an dieser Stelle (in Zeile 11) aus der Rolle der primären Sprecherin, indem sie ihre Antwort positiv evaluiert, ihre Antworterwartung aber aufrecht erhält und sich Norena zuwendet.

In dieser Zugfolge bereitet die Lehrerin den Wechsel zum Referenzraum Buchwelt vor und vollzieht später den Wechsel von der Buchwelt zum Weltwissen. Diese Bewegungen werden von der Lehrerin initiiert und von Alena adaptiv nachvollzogen. Alenas wiederholte Wechsel von der Buchwelt zur Metakommunikation erweisen sich als geeignete Strategien, um sich aus einem inhaltlich nicht einlösbaren Zugzwang zu befreien. Wechsel zwischen Referenzräumen müssen interaktiv markiert werden, damit sie von den AkteurInnen nachvollzogen werden können. Die Lehrerin markiert den ersten Wechsel mehrfach, indem sie Alena (praktisch handelnd) die Igel-Seite zeigt, mit dem Pronomen «das» («Möchtest du das sagen?») das Zeigen auch verbal vollzieht und ihr dann das Buch übergibt. Den zweiten Wechsel markiert sie paraverbal durch das Senken der Stimme und verbal durch die Formulierung «... wenn es gefährlich wird», die auf eine verallgemeinerte Situation, nicht auf eine Szene im Bilderbuch hinweist. Für die erste Zugfolge lässt sich damit festhalten, dass Wechsel von Referenzräumen (auch) diskursive Funktionen erfüllen (Befreiung aus einem nicht einlösbaren Zugzwang bzw. Unterstützung beim Erfüllen eines Zugzwangs) und dass sie mit verschiedenen Mitteln (praktisches Handeln mit Objekten, praktisches und verbales Zeigen, Variation der Prosodie) markiert werden.

Nachdem Norena von der Lehrerin das Rederecht als primäre Sprecherin erhalten hat, beantwortet sie (in Zeile 12) deren Frage (Was tut der Igel bei Gefahr?) mit einem konkretisierenden Beispiel: Wenn ein Auto kommt, rollt sich der Igel zu einem stacheligen Stein zusammen, so dass ihm das Auto nichts anhaben kann. Das Einrollen veranschaulicht sie mit Handbewegungen. Norena bleibt mit ihrer Antwort auf die Realität ausgerichtet, bezieht sich aber nicht auf abstraktes Weltwissen, sondern skizziert eine vorgestellte Situation. Damit vollzieht sie einerseits einen Wechsel des Referenzraums. Anderseits schliesst sie mit ihrer Antwort und durch die Verwendung eines (fingierten) Gegenstands an das Handeln der Lehrerin an. Die Lehrerin greift Norenas Handbewegung sofort auf und unterstützt sie beim Sprechen, indem sie den Stoffigel zu einer Kugel einrollt. Mit ihrem nächsten Zug (in Zeile 13) nimmt die Lehrerin mehrere Justierungen vor: Sie wechselt zurück zum Referenzraum des Weltwissens, sie evaluiert die Aussage von Norena teils kritisch-berichtigend (Einrollen schützt nicht vor Autos), teils positiv-bestätigend (Einrollen schützt vor anderen Feinden), und sie expliziert verbal und praktisch handelnd die kritische Differenz zwischen dem Stoff-Igel und seinem realen Referenzobjekt. 


\section{Zugfolge 2 der Igel-Eisode}

\begin{tabular}{|c|c|c|}
\hline Die LP rollt den Stoff-Igel zu einem Ball. & 12 & $\begin{array}{l}\text { Norena erklärt (und zeigt mit den Händen), dass } \\
\text { sich der Igel zu einem stachligen Stein einrolle } \\
\text { und ein Auto ihm nichts mehr anhaben könne. }\end{array}$ \\
\hline \multirow[t]{2}{*}{$\begin{array}{l}\text { Die LP blickt in die Runde und stellt Norenas } \\
\text { Antwort richtig: Autos seien gefährlich für den } \\
\text { Igel, auch wenn er eingerollt sei. Dabei hält den } \\
\text { Zeigefinger in die Höhe. } \\
\text { Sie bestätigt, dass er sich durch Einrollen vor } \\
\text { Feinden schützen könne. } \\
\text { Sie streichelt die weichen Stoffstacheln und } \\
\text { erklärt, dass richtige Igel stachliger seien. } \\
\text { Sie legt den Igel weg. }\end{array}$} & 13 & \\
\hline & 14 & $\begin{array}{l}\text { Norena wendet ein, dass der Igel mit seinen } \\
\text { Stacheln die Autoreifen zerstechen könnte. }\end{array}$ \\
\hline \multirow{3}{*}{$\begin{array}{l}\text { Die LP entgegnet zuerst, dass sie das nicht } \\
\text { wisse, und dann, dass sie das nicht glaube. } \\
\text { Sie wiederholt Norenas Aussage und bestärkt ihren } \\
\text { Zweifel daran. }\end{array}$} & 15 & \\
\hline & & \\
\hline & 16 & $\begin{array}{l}\text { Lisa verweist auf die Existenz von } \\
\text { Winterreifen. }\end{array}$ \\
\hline \multirow{2}{*}{$\begin{array}{l}\text { Die LP bestätigt Lisas Aussage schmunzelnd. } \\
\text { Sie steht auf und geht auf Alena zu. }\end{array}$} & 17 & \\
\hline & 18 & $\begin{array}{l}\text { Lisa berichtet, dass sie } \\
\text { Winterreifen zuhause hätten. } \\
\text { Alena hält der LP das Buch hin. }\end{array}$ \\
\hline $\begin{array}{l}\text { Die LP nimmt das Buch entgegen. } \\
\text { Sie wiederholt Lisas Aussage. } \\
\text { Sie berichtet von ihren eigenen } \\
\text { Winterreifen, die immer noch } \\
\text { montiert sind. }\end{array}$ & 19 & \\
\hline $\begin{array}{l}\text { Sie stellt sich vor, dass es plötzlich wieder schneien } \\
\text { könnte. } \\
\text { Sie blättert die Seite um. }\end{array}$ & 20 & \\
\hline
\end{tabular}

Abbildung 3: Igel-Episode, 2. Zugfolge. Schriftart Arial = auf Wissen über die Realität bezogen, Schriftart Times = auf vorgestellte Realität bezogen, Schriftart Courier = auf erlebte Realität bezogen; fett $=$ mit Objekten praktisch handelnd; unterstrichen = auf das gemeinsame Handeln im Hier-und-Jetzt bezogen

In der Folge (in Zeile 14) bezieht Norena diskursiv und inhaltlich eine Gegenposition zur Lehrerin: Sie wechselt zurück zu «ihrem» Referenzraum der vorgestellten Realität und verteidigt ihre frühere Aussage, der Igel könne sich durch Einrollen auch vor Autos schützen, wobei sie den Hinweis der Lehrerin auf die spitzen Stacheln geschickt in ihr Argument miteinbezieht. Die Lehrerin reagiert (in Zeile 15), indem sie Norenas Aussage inhaltlich negativ, aber wohlwollend und gesichtsbewahrend evaluiert: Sie weist sie zwar zurück, bezieht sich dabei aber nicht auf gesichertes Weltwissen, sondern auf ihre persönliche Einschätzung. 
Ausserdem folgt sie Norenas Wechsel zum Referenzraum der vorgestellten Realität. An dieser Stelle (in Zeile 16) bringt sich Lisa aus eigener Initiative ins Gespräch ein. Ihre Aussage ("Aber es gibt Winterreifen.») ist sprachformal und diskursiv als Gegenargument markiert, inhaltlich bestärkt sie aber die Position der Lehrerin. Diese Ambivalenz führt dazu, dass die Lehrerin (in Zeile 17) belustigt ist, Lisas Aussage nicht inhaltlich, sondern nur formal-abschliessend evaluiert und aufsteht, um (im Hier-und-Jetzt) das Buch von Alena zu holen und die Episode zu beenden. Lisa lässt aber noch nicht locker: Sie bleibt (in Zeile 18) bei ihrem Thema (Winterreifen) und wechselt in den neuen Referenzraum der erlebten Realität. Diesmal folgt ihr die Lehrerin: Sie anerkennt (in Zeile 19) Lisas Aussage und berichtet ebenfalls von ihrer eigenen Lebenswelt. Dann wechselt sie in den Referenzraum der vorgestellten Realität. Die Rückgabe des Bilderbuchs von Alena an die Lehrerin (in Zeilen 18 und 19) vollzieht sich beiläufig und wird nicht zum Gegenstand der geteilten Aufmerksamkeit. Der letzte, persönlich gefärbte Zug der Lehrerin suspendiert den formalen Abschluss der Episode.

In der zweiten Zugfolge dieser Episode geht es nur noch um die reale Welt, genauer: um Weltwissen, vorgestellte und erlebte Wirklichkeit. Die Wechsel zwischen diesen drei Referenzräumen werden von den Kindern initiiert und führen dazu, dass kurze, von der Lehrerin bereits abgeschlossene Diskurseinheiten variiert (Zeilen 14 und 18) oder verlängert werden (Zeile 16). Sie erfüllen damit eine gesprächselaborierende Funktion, die in diesem Beispiel ausschliesslich von den Kindern genutzt wird. Zudem kann das Beibehalten oder Wechseln von Referenzräumen von Zug zu Zug auch Ausdruck unterschiedlicher - adaptiv-nachvollziehender bzw. initiativ-steuernder Handlungsweisen sein und auf unterschiedliche Gesprächsrollen hinweisen. In dieser Funktion werden sie auch von der Lehrerin eingesetzt, als sie sich den von Norena und Lisa vorgenommenen Wechseln anschliesst (in Zeilen 15, 17 und 19) oder widersetzt (in Zeile 13). Markiert wird der Wechsel zum Weltwissen von der Lehrerin durch eine Geste (mit der Bedeutung "Aufpassen: Wichtig!») und durch die verallgemeinernde Formulierung "Autos sind gefährlich für den Igel.» Norena markiert den Referenzraum der vorgestellten Realität durch die Verwendung des Konjunktivs. Lisa wechselt zurück zum Indikativ, um ihr Weltwissen einzubringen (in Zeile 16), und danach vom unpersönlichen «es» zum persönlichen "wir", als sie sich auf ihr eigenes Erleben bezieht (in Zeile 18). Der Wechsel des Referenzraums erfüllt in dieser zweiten Zugfolge die diskursiven Funktionen der Elaboration sowie der Ausgestaltung der eigenen Gesprächsrolle, wird durch Gesten und spezifische sprachliche Mittel (Pronomina, Modi) markiert und v.a. von den Kindern initiiert.

\section{Bündelung der Auswertungsergebnisse}

Das Phänonem «Wechsel von Referenzräumen» konnte im Hinblick auf drei Aspekte konzeptionell ausgearbeitet werden: Erstens erfüllen solche Wechsel 
verschiedene diskursive Funktionen (Befreiung aus einem Zugzwang, Unterstützung beim Erfüllen eines Zugzwangs, Gesprächselaboration und Ausgestaltung von Gesprächsrollen). Zweitens werden sie mit unterschiedlichen Mitteln markiert (praktisches Handeln mit Objekten, Blickbewegungen, prosodische Variation, gestische Symbole und sprachliche Mittel wie deiktische Zeigewörter, Pronomina und Modi). Drittens machen neben der Lehrerin auch die Kinder aktiven Gebrauch von ihnen.

Daneben sind bei der sequenziellen Auswertung dieser Episode verschiedene andere Phänomene manifest geworden, die hier nicht ausführlich behandelt werden können, aber wenigstens skizziert werden sollen:

Das «offizielle» Unterrichtsprojekt der Lehrerin, das gemeinsame, schrittweise von ihr angeleitete Nacherzählen des Bilderbuchs im Kreis, wird zunächst von ihr selbst variiert (als sie von der Buchwelt zum Weltwissen wechselt und neben Alena auch Norena miteinbezieht) und danach durch die Initiativen von Norena und Lisa suspendiert. Danach nimmt die Lehrerin das Buch und die Führungsrolle wieder an sich, um das Unterrichtsprojekt fortzusetzen. Im Verlauf eines geführten und musterhaften Ablaufs öffnet sich aus der Situation heraus ein Gesprächsraum, der von den Kindern aktiv genutzt und von der Lehrerin während einer begrenzten Zeit zugelassen und mitbearbeitet wird.

Die erste Zugfolge ist exemplarisch für das Interaktionsmuster des schulischen Abfragens: Gesteuert und unterstützt von der Lehrerin soll Alena vor dem Publikum der Klasse im Dreischritt von Frage, Antwort und Evaluation gemeinsam erarbeitetes Wissen wiedergeben. In der zweiten Zugfolge übernehmen die Kinder die Initiative, während die Lehrerin sich vorwiegend adaptiv-nachvollziehend beteiligt. Dabei bleibt sie aber die «Drehscheibe» des Gesprächs: Die Züge der Kinder sind immer an sie gerichtet und werden von ihr beantwortet. Das Beispiel zeigt, dass die Asymmetrie, die für die Beziehung zwischen Lehrpersonen und Schülerinnen und Schülern konstituierend ist, in Gesprächen phasenweise aufgelockert werden kann, damit aber nicht aufgehoben ist.

In dieser kurzen Passage kommen vier verschiedene Sprachhandlungen vor, die direkt mit unterschiedlichen Referenzräumen in Beziehung stehen: das Nacherzählen mit der fiktionalen Buchwelt, das Berichten, Erklären und Argumentieren mit der Realität (als erlebte und vorgestellte Realität sowie Weltwissen). Zwischen den Referenzräumen und den Funktionen und Formen dieser Sprachhandlungen bestehen klare Bezüge, und der präzise Nachvollzug von Wechseln zwischen Referenzräumen dürfte beim Erwerb dieser anspruchsvollen Diskursfähigkeiten eine wichtige Rolle spielen.

Schliesslich zeigt das Beispiel von Alena, dass im Unterricht erarbeitetes Wissen, welches nicht direkt und situativ erlebt, sondern indirekt und medial (durch sprachliche und bildliche Repräsentationen) vermittelt wurde, nicht ohne Weiteres vorausgesetzt und von den Kindern als Gesprächsressource genutzt werden kann. Dagegen beteiligen sich die Kinder besonders aktiv an Gesprächen, wenn sie auf ihre eigenen thematischen Expertisen (Norena auf ihre Vorstellung, Lisa auf ihr Wissen und Erleben) zurückgreifen können. 
Der in diesem Abschnitt dargestellte Prozess der sequenziellen Auswertung und Konzeptualisierung von Phänomenen läuft zurzeit parallel zu den Erhebungen in vier weiteren Kindergartenklassen. Die systematische Ausarbeitung der Konzepte, ihre breitere empirische und theoretische Verankerung sowie die Erarbeitung von Fallportraits werden im weiteren Projektverlauf noch $\mathrm{zu}$ leisten sein.

\section{Verortung im Tagungsthema}

Rauschenbach (2007) strukturiert das Denkmodell des formalen und informellen Lernens anhand der Dimensionen Bildungsorte, Bildungsmodalitäten und Bildungsinhalte (Rauschenbach 2007). Im Projekt ProSpiK wird der formale Bildungsort Kindergarten untersucht. Aufgrund der bisherigen Auswertungen ist bereits klar, dass hier formale und informelle Bildungsprozesse stattfinden und sowohl curriculare als auch nicht-curriculare Bildungsinhalte vermittelt und erworben werden. $\mathrm{Zu}$ den formal vermittelten curricularen Inhalten gehören etwa das Einhalten von Gesprächsregeln oder das (Nach-) Erzählen von Geschichten (D-EDK, 2013). Informell erworben werden zum Beispiel die diskursiven Fähigkeiten, zwischen Referenzräumen zu wechseln, verschiedene Sprachhandlungen zu realisieren oder Gesprächsrollen situationsgemäss auszugestalten. Diese Befunde unterstreichen die Funktion des Kindergartens, möglichst allen Kindern frühzeitig Zugang zu jener Alltagsbildung zu verschaffen, die in der Schule stillschweigend vorausgesetzt wird, aber nicht in allen Familien gleichermassen präsent ist (Lahire, 1993).

Um eine solche «rationale Pädagogik» (Bourdieu \& Passeron 1971, S. 88) im Kindergarten systematisch zu verankern, müssen Lehrpersonen allerdings entsprechend aus- und weitergebildet werden. Dabei könnte es erstens darum gehen, ihr Verständnis des Kindergartens als Ort des Übergangs von informellen $\mathrm{zu}$ formalen Bildungsprozessen und von nicht-curricularen $\mathrm{zu}$ curricularen Bildungsinhalten zu vertiefen. Aus dieser Perspektive müsste die bildungssprachliche Förderung der Kinder individuell zugeschnitten sein und graduell verlaufen, um mit den schulischen Bildungsprozessen an die vorschulische Alltagsbildung anzuschliessen. Zweitens müssten Kindergartenlehrpersonen für die Mikroebene von Bildungsprozessen sensibilisiert und bei der Weiterentwicklung ihres interaktiven Handelns unterstützt werden. Drittens wäre es wichtig, bei den Lehrpersonen ein Bewusstsein für die zentrale Bedeutung des sprachlichen Handelns zu schaffen und sie beim Aufbau eines praktischen Handlungswissens für die Förderung bildungssprachlicher Fähigkeiten zu unterstützen. Der Einsatz von Videofeedbacks in der Aus- und Weiterbildung von Lehrpersonen dürfte ein viel versprechender Weg sein, um mit Kindergarten-Lehrpersonen in einer neuen Verbindlichkeit an ihrem Handeln und ihren Orientierungen bei der formalen und informellen Sprachförderung zu arbeiten. 


\section{Anmerkungen}

1 Dieses Projekt wird vom Schweizerischen Nationalfonds gefördert und vom September 2012 bis August 2015 durchgeführt.

2 Im Tessin und (in unterschiedlicher Ausprägung) in der Romandie sind die öffentlichen Einrichtungen für 4- bis 6-jährige Kinder schon länger als schulische Bildungsorganisationen ausgestaltet (Sörensen \& Thévenaz-Christen, 2003).

3 Studien, die Diskursfähigkeiten von Kinder in Gesprächen mit Forschenden untersuchen, werden nicht berücksichtigt, weil es hier um das Handeln der pädagogischen Fachpersonen in Interaktionen geht.

4 Globale Zugzwänge entstehen, wenn Kinder aufgefordert werden, «mündliche Texte» im Sinne von längeren monologischen Gesprächsbeiträgen zu produzieren. Dazu gehören Sprachhandlungen wie Erzählen, Erklären oder Berichten.

5 Gute Leserinnen und Leser verfügen nicht nur über automatisierte Prozesse der Schriftund Sprachverarbeitung, sondern auch und in besonderem Masse über hierarchiehöhere, flexible Prozesse des Textverstehens (Schnotz \& Dutke, 2004). Die Entwicklung dieser basalen und hierarchiehöheren Fähigkeiten setzt bereits im Vorschulalter ein und verläuft relativ unabhängig (Whitehurst \& Lonigan, 2001). Bei der frühen literalen Förderung muss deshalb auch der Erwerb textueller und pragmatischer Fähigkeiten gezielt unterstützt werden.

6 Eine detailliertere Darstellung der Bezugstheorien findet sich in Isler \& Künzli (2011).

7 Eine detailliertere Darstellung der methodologischen Grundlagen findet sich in Isler \& Künzli (2011).

8 Diese Sequenz wurde aus dem Datenkorpus ausgewählt, weil hier eine schultypische kommunikative Form («Nacherzählen») unter Beteiligung der Lehrerin über etliche Züge hinweg interaktiv realisiert wird.

9 Diese paraphrasierende Darstellung dient der leichteren Lesbarkeit in Publikationen. Die Auswertungen basieren auf Videodaten und Transkripten, nicht auf Paraphrasierungen. Die Originaltranskripte sind für Forschende auf Anfrage bei uns einsehbar. Alle Kindernamen wurden geändert.

\section{Literaturverzeichnis}

Aellig, S., Alt, E. \& Bass, S. (2009). Lezus - von der Lauterfassung zur Schrift. Bern: Schulverlag plus.

Baumann Schenker, S., Knechtel, N. \& Kruse, G. (2010). Parcours Sprache. Bern: Schulverlag Plus.

Bildungsdirektion Kanton Zürich (2008). Lehrplan für die Kindergartenstufe des Kantons Zürich. Zürich: Lehrmittelverlag Zürich.

Bitter Bättig, F, Büchel, E, Eriksson, B, Gyger, M, Landert K, Schader, B. \& Stamm, G. (2012). Einschätzungsraster Erstsprache Deutsch 4bis8. Bern. Schulverlag plus.

Bos, W., Lankes, E., Schwippert, K., Valtin, R., Voss, A., Badel, I. \& Plassmeier, N. (2003). Lesekompetenzen deutscher Grundschülerinnen und Grundschüler am Ende der vierten Jahrgangsstufe im internationalen Vergleich. In W. Bos, E. Lankes \& K. Schwippert (Hrsg.), Erste Ergebnisse aus IGLU (S. 69-142). Münster: Waxmann.

Bourdieu, P. (2001). Die konservative Schule. Die soziale Chancenungleichheit gegenüber Schule und Kultur. In M. Steinrücke (Hrsg.). Wie die Kultur zum Bauern kommt. Über Bildung, Schule und Politik (S. 25-52). Hamburg: VSA.

Bourdieu, P. \& Passeron, J. (1971). Die Illusion der Chancengleichheit. Untersuchungen zur Soziologie des Bildungswesens am Beispiel Frankreichs. Stuttgart: Klett.

Bruner, J. (1983 [2002]). Wie das Kind sprechen lernt. Bern: Hans Huber.

CIIP (2008). Plan d'édtude romand. Neuchâtel: Conférence intercantonale de l'instruction publique de la Suisse romande et du Tessin. 
D-EDK (2013). Lehrplan 21: Sprachen (Konsultationsfassung). Luzern: Deutschschweizer Erziehungsdirektoren-Konferenz.

EDK (2013). Effektive Besuchsdauer (Vorschule). Kantonsumfrage. Schuljahr 2012 / 2013. http://www.edk.ch/dyn/15332.php (abgerufen am 21.1.2014).

Ehlich, K. (2005). Sprachaneignung und deren Feststellung bei Kinder mit und ohne Migrationshintergrund - was man weiss, was man braucht, was man erwarten kann. In K. Ehlich et al. (Hrsg.), Anforderungen an Verfahren der regelmässigen Sprachstandsfeststellung als Grundlage für die frühe und individuelle Förderung von Kindern mit und ohne Migrationshintergrund (S. 11-63). Berlin: Bundesministerium für Bildung und Forschung.

Erard, S. \& Schneuwly, B. (2005). Le didactique de l'oral: savoirs ou compétences? In J.-P. Bronckart, E. Bulea \& M. Pouliot (Éd.). Repenser l'enseignement des langues: comment indetifier et exploiter les compétences? (pp. 69-97). Villeneuve d'Ascq cedex: Presses Universitaires du Septentrion.

Erziehungsdirektion des Kantons Bern (1999). Lehrplan Kindergarten für den deutschsprachigen Teil des Kantons Bern. Bern: Erziehungsdirektion des Kantons Bern.

Farran, D., Aydogan, C., Kang, S. \& Lipsey, M. (2006). Preschool Classroom Environments and the Quantity and Quality of Children's Literacy and Language Behaviours. In D. Dickinson \& D. Neuman (Eds.), Handbook of Early Literacy Research, Volume 2 (pp. 257-268). New York: The Guilford Press.

Flick, U. (2000 [2008]). Triangulation in der qualitativen Forschung. In U. Flick, E. von Kardorff \& I. Steinke (Hrsg.), Qualitative Forschung. Ein Handbuch (S. 309-318). Reinbek bei Hamburg: Rowohlt.

Fried, L. (2008). Pädagogische Sprachdiagnostik für Vorschulkinder - Dynamik, Stand und Ausblick. In Roßbach, H. \& Rossfeld, H. (Hrsg.): Frühpädagogische Förderung in Institutionen (S. 63-78). Wiesbaden: VS Verlag für Sozialwissenschaften (Zeitschrift für Erziehungswissenschaft, Sonderheft 22 / 2008).

Fried, L. (2010). Sprachliche Bildung. In M. Stamm \& D. Edelmann (Hrsg.), Frühkindliche Bildung, Betreuung und Erziehung (S. 155-175). Zürich: Rüegger.

Garfinkel, H. (1967). Studies in Ethnomethodology. Englewood Cliffs: Prentice Hall.

Gasteiger-Klicpera, B., Knapp, W. \& Kucharz, D. (2010). Abschlussbericht der Wissenschaftlichen Begleitung des Programms «Sag mal was - Sprachförderung für Vorschulkinder». Weingarten: sPäd. Hochschule Weingarten. Verfügbar unter http://www.ph-weingarten. de/zep/Projekte (abgerufen am 23.2.2011).

Grossenbacher, S. (2007). Vorschulstufe: Vom Stiefkind zum «shooting star» im Bildungswesen? Referat anlässlich der Tagung «Erziehung und Bildung 4 bis 8», Kartause Ittingen, 21.6. 2007. Verfügbar unter http://edk-ost.ch/Donnerstag-21-Juni-2007.147.0.html (abgerufen am 8.7.2010).

Habermas, J. (1968). Erkenntnis und Interesse. Frankfurt am Main: Suhrkamp.

Heath, S. (1983). Ways with words. Language, life, and work in communities and classrooms. Cambridge: Cambridge University Press.

Heller, V. (2012). Kommunikative Erfahrungen von Kindern in Familie und Unterricht. Passungen und Divergenzen. Tübingen: Stauffenburg.

Henry, A. \& Pianta, R. (2011). Effective Teacher-Child Interactions and Childrens Literacy: Evidence for Scalable, Aligned Approaches to Professional Development. In S. Neuman \& D. Dickinson (Eds.), Handbook of Early Literacy Research, Volume 3 (pp. 308-321). New York: Guilford Press.

Isler, D. \& Künzli, S. (2011). Untersuchung sprachlicher Praktiken in Familien und im Kindergarten: Methodologische und theoretische Grundlagen. Schweizerische Zeitschrift für Bildungswissenschaften, 33 (2), 191-210.

Isler, D. (2014). Vorschulischer Erwerb von Literalität in Familien. Université de Genève (Dissertation). Online zugänglich unter http://archive-ouverte.unige.ch/unige:40025. 
Käsler, D. (2005). Sprachbarrieren im Bildungswesen. In P. Berger \& H. Kahlert (Hrsg.), Institutionalisierte Ungleichheiten. Wie das Bildungswesen Chancen blockiert (S. 130-154). Weinheim/München: Juventa.

Knoblauch, H. (2006). Videography. Focused Ethnography and Video Analysis. In H. Knoblauch, B. Schnettler, J. Raab, H. Soeffner (Ed.), Video Analysis. Methodology and Methods. Qualitative Audiovisual Data Analysis in Sociology (pp. 69-83). Frankfurt a. M.: Peter Lang.

Knoblauch, H. (2013). Grundbegriffe und Aufgaben des kommunikativen Konstruktivismus. In R. Keller, H. Knoblauch \& J. Reichertz (Hrsg.), Kommunikativer Konstruktivismus. Theoretische und empirische Arbeiten zu einem neuen wissenssoziologischen Ansatz (S. 25-47). Wiesbaden: Springer.

Koch, P. \& Oesterreicher, W. (1994). Schriftlichkeit und Sprache. In: H. Günther \& O. Ludwig (Hrsg.), Schrift und Schriftlichkeit, Band 1 (S. 587-604). Berlin: De Gruyter.

König, A. (2010). Videoanalyse als Möglichkeit, Prozessqualität differenziert zu erfassen: Eine Untersuchung zur interaktionalistischen Lernumwelt im Kindergarten. In M. Stamm \& D. Edelmann (Hrsg.), Frühkindliche Bildung, Betreuung und Erziehung (S. 335-357). Zürich: Rüegger.

Kuger, S. \& Kluczniok, K. (2008). Prozessqualität im Kindergarten - Konzept, Umsetzung und Befunde. In H. Roßbach \& H. Rossfeld (Hrsg.), Frühpädagogische Förderung in Institutionen (S. 159-177). Wiesbaden: VS Verlag für Sozialwissenschaften (Zeitschrift für Erziehungswissenschaft, Sonderheft 22 / 2008).

Künzli, S. \& Isler, D. \& Leemann, R. (2010). Frühe Literalität als soziale Praxis - Analyse von Mikroprozessen der Reproduktion von Bildungsungleichheit. Zeitschrift für Soziologie der Erziehung und Sozialisation, 30 (1), 60-73.

Lahire, B. (1993). Culture écrite et inégalités scolaires. Lyon: Presses universitaires de Lyon.

Morek, M. (2012). Kinder erklären. Interaktionen in Familien und Unterricht im Vergleich. Tübingen: Stauffenburg.

Moser, U. (2005). Lernbedingungen in Schulklassen zu Beginn der 1. Klasse. In U. Moser \& M. Stamm \& J. Hollenweger (Hrsg.), Für die Schule bereit? Lesen, Wortschatz, Mathematik und soziale Kompetenzen beim Schuleintritt. (S. 167-185). Oberentfelden: Sauerländer.

Moser, U. \& Berweger, S. (2008): Wortgewandt und zahlenstark. Zürich: Lehrmittelverlag Zürich.

Niedermann, A. \& Sassenroth, M. (2002). Lesestufen. Ein Instrument zur Feststellung und Förderung der Lesenetwicklung. Zug: Klett und Balmer.

Niklas, F., Schmiedeler, S. \& Schneider, W. (2010). Heterogenität in den Lernvoraussetzungen von Vorschulkindern. Zeitschrift für Grundschulforschung. Bildung im Elementarund Primarbereich, 3. Jg. (2010), Heft 1, 18-31.

OECD (2010). PISA 2009. Results: Overcoming Social Background - Equity in Learning Opportunities and Outcomes (Volume II) http://dx.doi.org/10.1787/9789264091504-en (abgerufen am 1.2.2011).

Quasthoff, U. \& Kern, F. (2007). Familiale Interaktionsmuster und kindliche Diskursfähigkeit: Mögliche Auswirkungen interaktiver Stile auf diskursive Praktiken und Kompetenzen bei Schulkindern. In H. Hausendorf (Hrsg.), Gespräch als Prozess. Linguistische Aspekte der Zeitlichkeit verbaler Interaktion (S. 277-305). Tübingen: Gunter Narr.

Quasthoff, U., Fried, L., Katz-Bernstein, N., Lengning, A., Schröder, A. \& Stude, J. (2011). (Vor-)Schulkinder erzählen im Gespräch. Kompetenzunterschiede systematisch erkennen und fördern. Baltmannsweiler: Schneider.

Rauschenbach, T. (2007). Im Schatten der formalen Bildung. Alltagsbildung als Schlüsselfrage $\operatorname{der}$ Zukunft. Diskurs Kindheits- und Jugendforschung 4-2007, 439-453.

Roßbach, H., Klugcniok, K. \& Kuger, S. (2008). Auswirkungen eines Kindergartenbesuchs auf den kognitiv-leistungsbezogenen Entwicklungsstand von Kindern. In H. Roßbach \& 
H. Blossfeld (Hrsg.), Frühpädagogische Förderung in Institutionen. Zeitschrift für Erziehungswissenschaft, Sonderheft 22, 139-158.

Sammons, P., Anders, Y., Sylva, K., Melhuish, E., Siraj-Blatchford, I., Taggart, B. \& Barreau, S. (2008). Children's Cognitive Attainment and Progress in English Primary Schools During Key Stage 2: Investigating the potential continuing influences of pre-school education. In H. Roßbach \& H. Blossfeld (Hrsg.), Frühpädagogische Förderung in Institutionen. Zeitschrift für Erziehungswissenschaft, Sonderheft 22, 179-197.

Schnotz, W. \& Dutke, S. (2004). Kognitionspsychologische Grundlagen der Lesekompetenz: Mehrebenenverarbeitung anhand multipler Informationsquellen. In U. Schiefele, C. Artelt, W. Schneider \& P. Stanat (Hrsg.), Struktur, Entwicklung und Förderung von Lesekompetenz (S. 61-99). Wiesbaden: VS Verlag.

Stude, J. (2013). Kinder sprechen über Sprache. Eine Untersuchung zu interaktiven Ressourcen des frühen Erwerbs metasprachlicher Kompetenzen. Stuttgart: Fillibach bei Klett.

Schütz, A. (1932 [1984]). Der sinnhafte Aufbau der sozialen Welt. Frankfurt am Main: Suhrkamp.

Sörensen B. \& Thévenaz-Christen, T. (2003 ). Editorial zum Themenschwerpunktheft "Kleinkinder- und Vorschulbildung: Praktiken und Ziele». Schweizerische Zeitschrift für Bildungswissenschaften, 25 (2), 207-210.

Stamm, M. (2012). Qualität und frühkindliche Bildung. Grundlagen und Perspektiven für die Qualitätsentwicklung von vorschulischen Angeboten. Dossier 12/3. Fribourg: Departement Erziehungswissenschaften der Universität Fribourg.

Strauss, A. (1990 [1994]). Grundlagen qualitativer Sozialforschung. Datenanalyse und Theoriebildung in der empirischen und soziologischen Forschung. München: Fink.

Strauss, A. \& Corbin, J. (1996). Grounded Theory: Grundlagen Qualitativer Sozialforschung. Weinheim: Beltz (PVU).

Sylva, K., Chan, L., Melhuish, E., Sammons, P., Siraj-Blatchford, I. \& Taggart, B. (2011). Emergent Literacy Environments: Home and Preschool Influences on Children's Literacy Development. In S. Neuman, \& D. Dickinson (Eds). Handbook of Early Literacy Research, Volume 3 (pp. 97-117). New York: Guilford Press.

Thévenaz-Christen, T. (2005). Les prémices de la forme scolaire. Etudes d'activités langagières orales à l'école enfantine genevoise. Unveröffentlichte Dissertation, Université de Genève.

Thole, W., Göbel, S. \& Milbrandt, B. (2013). Kinder und Kindheiten im Blick unterschiedlicher Fachkulturen. In M. Stamm \& D. Edelmann (Hrsg.), Handbuch frühkindliche Bildungsforschung. (S. 23-36). Wiesbaden: Springer VS.

Tomasello, M. (1999). The Cultural Origins of Human Cognition. Cambridge: Harvard University Press.

Vasilyeva, M. \& Waterfall, H. (2011). Variability in Language Development: Relation to Socioeconomic Status and Environmental Input. In S. Neuman \& K. Dickinson (Eds.), Handbook of Eraly Literacy Research. Vol. 3 (pp. 36-48). New York: Guilford Press.

Weinert, S., Ebert, S. \& Dubowy, M. (2010). Kompetenzen und soziale Disparitäten im Vorschulalter. Zeitschrift für Grundschulforschung. Bildung im Elementar- und Primarbereich, 3. Jg. (2010), Heft 1, 32-45.

Whitehurst, G. \& Lonigan, C. (2001). Emergent Literacy: Development from Prereaders to Readers. In S. Neumann \& D. Dickinson (Eds.), Handbook of Eraly Literacy Research. Vol. 1 (pp. 11-29). New York: Guilford Press.

Witzig, H. (2002). Geschichte des Kindergartens. In C. Walter \& K. Fasseing (Hrsg.), Kindergarten. Grundlagen aktueller Kindergartendidaktik (S. 15-31). Winterthur: ProKiGa-Lehrmittelverlag.

Wygotski, L. (1934 [1986]). Denken und Sprechen. Frankfurt a. M.: Fischer. 
Schlagworte: Sprachliche Bildungsprozesse, Bildungsungleichheit, Kindergarten, formales und informelles Gespräch, Interaktionsanalysen

\title{
Discussions au quotidien à l'école enfantine: structures informelles pour l'acquisition d'aptitudes langagières scolaires
}

\section{Ré sumé}

La fréquentation d'une institution de formation préscolaire qualifiée peut contribuer à augmenter les chances des enfants défavorisés. A partir de ce constat, le projet ProSpiK s'attache à étudier les processus de promotion langagière dans des écoles enfantines en Suisse alémanique. Il met en particulier l'accent sur l'action des enseignants lors de discussions formelles ou informelles au quotidien avec les enfants. Des interactions vidéo filmées sont analysées par séquences afin de reconstruire la forme et les caractéristiques communicationnelles de leur processus de production. L'article présente les bases et la structure de ce projet. La procédure d'évaluation séquentielle et de développement conceptuel est ensuite présentée à partir d'un exemple concernant le concept "changement des espaces de référence». Il en ressort que des processus de formation aussi bien formels qu'informels ont lieu dans les discussions quotidiennes.

Mots-clés: Processus de formation langagière, égalité devant la formation, école enfantine, discussions formelles et informelles en classe, analyse d'interactions

\section{Linguaggio quotidiano alla scuola dell'infanzia - opportunità informali per l'acquisizione di competenze linguistiche scolastiche}

\begin{abstract}
Una formazione di qualità alla scola dell'infanzia contribuisce al miglioramento delle opportunità formative degli allievi e riduce le diseguaglianze formative. Nel progetto ProSpik sono stati analizzati i processi di promozione linguistica nelle scuole dell'infanzia della Svizzera tedesca. I ricercatori hanno posto attenzione al comportamento dei docenti negli scambi linguistici quotidiani con i bambini. Gli scambi, documentati tramite video, sono stati analizzati tramite procedure di analisi delle interazioni per identificare le loro forme caratteristiche (pattern) e i loro processi di produzione. L'articolo presenta il quadro teorico e l'impianto metodologico del progetto. Inoltre, la procedura di analisi delle sequenze e di formulazione delle classificazioni viene illustrata tramite l'esempio del concetto "cambiamento degli ambiti di riferimento». I risultati mostrano come nel linguaggio quotidiano di realizzino processi di apprendimento linguistico sia formali che informali.
\end{abstract}


Parole-chiave: Processi di apprendimento linguistico, linguaggio scolastico, diseguaglianze formative, scuola dell'infanzia, ricerca didattica, analisi delle interazioni.

\section{Everyday talk in Kindergarten classrooms: Informal opportunities for academic language acquisition}

\section{Summary}

High quality preschool education can contribute to the reduction of social inequality in education. The project ProSpiK therefore explores processes of language promotion and acquisition in Swiss Kindergarten classrooms. The focus is set on teachers actions during formal and informal talk with children. Videotaped interactions are scrutinized using sequential analysis procedures in order to reconstruct "communicative forms» (i.e. patterns) and the processes of their production. The article first presents the foundation and the design of the project. In addition, the procedure of sequential analysis and the conceptualisation of findings are exemplified with respect to the concept «change between referential spaces». The results emphasize the occurrence of both formal and informal processes of language promotion and acquisition during everyday talk in Kindergarten classrooms.

Keywords: Processes of language promotion and acquisition, academic language, inequality in education, Kindergarten, classroom formal and informal discussions, interactional analysis 
